\title{
Addition of Neostigmine and Tramadol to $1.5 \%$ Lidocaine for paracervical block to reduce post-operative pain in colporrhaphy
}

\author{
Alireza Kamali (1), Maryam Shokrpour (2), Bijan Yazdi (1), Atefeh Khalilpour (2) \\ (1) Department of Anesthesiology, Arak University of Medical Sciences, Arak, Iran; \\ (2) Department of Gynecology, Arak University of Medical Sciences, Arak, Iran
}

This article is distributed under the terms of the Creative Commons Attribution Noncommercial License (CC BY-NC 4.0) which permits any noncommercial use, distribution, and reproduction in any medium, provided the original author(s) and source are credited.

\begin{abstract}
Pain is a complex and multi-faceted human perception and several factors could dampen the pain. Therefore, we aimed to comparison of addition of neostigmine and tramadol to $1.5 \%$ lidocaine with paracervical block in reduce of post-operative pain in colporrhaphy. This study was a randomized and double-blind clinical trial for 108 patients' as candidate for colporrhaphy. Patients were randomly divided in three groups (Neostigmine, tramadol and control).We recorded pain in 2 and 6 and 12 hours after surgery, duration of analgesia and mean of use narcotic drug in 24 hours after surgery for all patients. Mean of narcotic drug used in 24 hours after surgery in neostigmine group was more than tramadol group $(\mathrm{p}=0 / 01)$.Pain in 2 and 6 and 12 hours after surgery in neostigmine group was more than tramadol group $(\mathrm{p}=0 / 01)$. Duration of analgesia in tramadol group was over neostigmine group and also, it was in neostigmine group was more than placebo $(\mathrm{p}=0 / 01)$. Taken together, tramadol could reduce pain in 2 and 6 and 12 hours after surgery and mean of narcotic drug used in 24 hours after surgery and increase duration of analgesia.
\end{abstract}

Key Words: Colporrhaphy, Neostigmine, Tramadol, pain after surgery

Eur J Transl Myol 28 (2): 185-192, 2018

Pelvic organ prolapse is referred to a state of pelvic organs drop that cause vaginal lump. Related factors include age, frequency of delivery, hormone deficiency, intense physical activity, constipation and chronic cough. ${ }^{1}$ Prolapse causes symptoms such as vaginal protrusion, painful sexual intercourse, lower back pain, sexual dysfunction, urinary incontinence, hard urination and hard bowel movement. ${ }^{2}$ Millions of women worldwide have undergone colporrhaphy and this is known as a global benchmark in women's health. In America, more than 300,000 surgeries in pelvic organ prolapse are performed. ${ }^{3}$ Colporrhaphy is a vaginal wall repair surgery. Vaginal wall defect includes cystocele (the prolapsed bladder into the vagina) and rectocele (prolapsed rectum in the vagina). Colporrhaphy includes two approaches: anterior (to treat cystocele or ureterocele) and posterior (to treat rectocele). ${ }^{4}$ Colporrhaphy is a common surgery on women. Anesthesia method in colporrhaphy depends on the surgery and the patient's condition, i.e., local, regional or general anesthesia. Anesthesiologists must adopt approaches that has maximum safety and comfort for the patient and the selected method of anesthesia should provide the best conditions for surgery. ${ }^{5}$ Pain is a routine postoperative side effect, whose control after surgery is an important objective of anesthesiologists. ${ }^{6}$ Although pain control during surgery is the primary objective, new analgesic methods aim to control pain after surgery. Postoperative pain control leads to patient satisfaction, reduced hospitalization and cost of treatment. ${ }^{7}$ A variety of therapies are available for treatment of postoperative pains. They include systemic analgesia techniques (opioids and non-opioids) and regional analgesia. ${ }^{8}$ Pain is a complex and multi-faceted human perception and several techniques could dampen the pain, one of the most important being regional analgesia. ${ }^{9,10}$ Its advantages are that the medications are not injected directly into the vein and thus the drugs are not delivered to vital organs, reducing complications. ${ }^{11}$ One of the available methods is paracervical block, that prevents transmission of pain along the sensory sympathetic and para-sympathetic fibers at the level of the entry of nerves into the uterus, at the surface of cervix inner canal. ${ }^{12}$ During cervical dilation, pain signal is transmitted by the parasympathetic fibers that are in keeping with uterine arteries and cardinal 
ligaments. That's why paracervical block is done at hours 3 and $9 .{ }^{13}$ However, since it has no effect on nerve of the upper part of the uterus, it cannot completely eliminate pain. ${ }^{14}$ In theory, it seems that the injection of an anesthetic into the uterus can block nerve terminals of body and fundus in the uterus and decrease pain more than paracervical block alone. ${ }^{15}$ Thus, the injection of anesthetic into the uterine cavity to reduce the pain resulting from dilatation seems reasonable. Lidocaine is a drug that is widely used in local anesthesia, it is cheap and its effect is fast. ${ }^{12}$ Lidocaine has fewer side effects than other local anesthetic drugs. Side effects of lidocaine in high doses are tremor and seizures and in case of hypersensitivity to the drug, allergic symptoms, in rare cases, bradycardia and hypotension. ${ }^{16}$ Drug supplements to the Lidocaine increase duration of analgesia and anesthesia and reduce postoperative complications. ${ }^{17}$ Neostigmine is a supplement that increase the duration of analgesia by affecting N- methyl-D-aspartate (NMDA) receptors. ${ }^{18,19}$ Tramadol is a synthetic drug that imitates the $\mu$ receptor and inhibits the reuptake of serotonin and norepinephrine. Its benefits in relieving postoperative pain include prevention of respiratory depression, demage of other major organs and gastrointestinal motility. Its common side effects $(6.1-1.6 \%)$ include dizziness, drowsiness, sweating, nausea, vomiting, dry mouth and headache. It should be taken cautiously in patients with epilepsy or increased intracranial pressure and it should not be taken in consumers of monoamine oxidase. $^{20,21}$. Hence, we tested combination of tramadol and neostigmine with lidocaine.

\section{Materials and Methods}

This study is a randomized, double-blind clinical trial on 108 colporrhaphy candidates referred to Taleghani Hospital in Arak (Iranian Registry of Clinical Trial with the number: IRCT.ARAKMU.REC.1394.298). In this study, patients who had the inclusion criteria and had the informed consent for their inclusion in the study were examined as study population. After admitting, 108 patients were randomly divided into three groups by random cube sampling: neostigmine, tramadol and control. In neostigmine group, $1.25 \mathrm{mg}$ neostigmine (equivalent to $0.5 \mathrm{ml}$ ) was added to $1.5 \%$ lidocaine. In tramadol group, $25 \mathrm{mg}$ tramadol (equivalent to $0.5 \mathrm{ml}$ ) was added to $1.5 \%$ lidocaine. In the control group, the same amount (equal to $0.5 \mathrm{ml}$ ) of normal saline was added to $1.5 \%$ lidocaine. Patients were prepared for colporrhaphy surgery, but first necessary monitoring oxygen saturation percent, electrocardiography (ECG), heart rate, respiration, temperature, non-invasive blood pressure, ET $\mathrm{CO}_{2}$ were done for patients, then, patients received 3-5 $\mathrm{mg}$ per $\mathrm{kg}$ of Ringer as cardiovascular expansion through a vein and were prepared for surgery. They were anesthetized by using $2 \mathrm{mg} / \mathrm{kg}$ of fentanyl and 2-3 mg of midazolam and 2-4 $\mathrm{mg} / \mathrm{kg}$ propofol and about $1-2 \mathrm{mg} / \mathrm{kg}$ atracurium induction. Then the patient is ventilated and then orally intubated and placed under anesthesia machine. After ensuring the right place of the tracheal tube and its fixation, surgery is allowed. During the surgery, patients received maintenance drug for general anesthesia through Isoflurane 1-1.5 minimum alveolar concentration (MAC). All the patients were monitored during anesthesia by basic monitoring. After surgery, patients were prepared for paracervical block at the end after being in proper position and 2 blocks were done by a gynecologist (supervisor) at 3 and 9 through syringe needle. All patients undergone paracervical block by one person (supervisor). The responsible intern observed all the blocks in all stages. Drugs used in paracervical block were prepared by the anesthesiologist (supervisor) for patients and was available for the responsible intern who was not aware of the content of the syringe and completed the questionnaires. So the gynecologist performing the block, intern, and patients were unaware of the drugs used in paracervical block. $1.5 \%$ lidocaine was used for all patients as the basic drug for paracervical block. In neostigmine group, $1.25 \mathrm{mg}$ neostigmine (equivalent to $0.5 \mathrm{cc}$ ), in tramadol group, 25 $\mathrm{mg}$ tramadol (equivalent to $0.5 \mathrm{ml}$ ) and in the control group, the same amount (equal to $0.5 \mathrm{ml}$ ) normal saline were added as a placebo. In all groups, the total volume of injected drugs in paracervical block reached up to $5 \mathrm{cc}$, so that all syringes used in paracervical block were equal for patients. For all these patients, $2 \mathrm{mg}$ midazolam and $2 \mathrm{mg} / \mathrm{kg}$ fentanyl were given as sedative drug that were injected for the patents after doing the block and then 5 $\mathrm{ml}$ of oxygen was given to each patient by face mask. In the case of failure of the block for these patients, they were undergone general anesthesia and were excluded from the research project. Once doing the paracervical block, patients' vital signs, including oxygen saturation, $\mathrm{ECG}$, heart rate, respiration, temperature, non-invasive blood pressure were recorded in the questionnaire. During surgery, blood pressure and heart rate were recoded every 15 minutes and finally at the end of surgery. Upon the arrival of patients to the recovery, in addition to vital signs, patients' pain scores were recorded in the questionnaire. Pain intensity was recorded through visual analogue scale at 2, 6 and $12 \mathrm{~h}$ after surgery by the intern. The average duration of analgesia for patients based on requesting the first pain reliever received by the patients and other demographic data as well as blood pressure, heart rate and mean pain reliever intake within 24 hours after surgery were recorded for each patient. Finally, the data obtained from questionnaires were analyzed by SPSS 19 and presented as statistical table chart.

\section{Inclusion criteria}

Anesthesiologists (ASA I and II), all colporrhaphy candidate women referred to Taleghani Hospital in Arak in the age group 30-55 years; all patients patients who undersigned the informed consent to enter the project. 
Table1. Comparison of the mean age of the patients undergoing Colporrhaphy, in the three groups of Tramadol, Neostigmine and placebo.

\begin{tabular}{lllll}
\hline Group & Tramadol & Neostigmine & placebo & P value \\
\hline Mean \pm SD & $41.9 \pm 4.8$ & $42.1 \pm 3.9$ & $41.7 \pm 3.1$ & $\mathrm{P} \geq 0.05$ \\
\hline
\end{tabular}

\section{Exclusion criteria}

1. Women outside the age group 30-55 years,

2. Patients in ASA classes 3 and 4,

3. All patients that paracervical block are not successful in them,

4. Patients without informed consent,

5. All patients whose surgery takes more than 90 minutes $\mathrm{BMI} \geq 35-6$.

Sampling and sample size

Each group 36 people for a total of 108 people.

$$
\begin{gathered}
N=\frac{\left(Z_{1-\frac{\alpha}{2}}+Z_{1-\beta}\right)^{2}\left(\delta_{1}+\delta_{2}\right)^{2}}{\left(\mu_{1}-\mu_{2}\right)^{2}} \\
\mu_{1}=2.5 \quad \delta_{1}=1.3 \quad Z_{1-\frac{\alpha}{2}}=1.96 \\
\mu_{2}=2 \quad \delta_{2}=0.6 \quad Z_{1-\beta}=2.33
\end{gathered}
$$

Information of all patients will be confidential for the project executor. All ethical statements will be considered in all stages of research following the Helsinki recommendations. The trial was approved by the the research committees of bioethics of Arak University of Medical Sciences. Code of ethics: (ir.arakmu.rec.1394.298). Registration code of Iran clinical trial center: IR2016050320258n7.

\section{Results and Discussion}

Patients were randomly divided into 3 groups: neostigmine, tramadol and control.

The average age of all three groups was 41 years old (Table 1.) and there was no significant difference between the 3 groups in terms of age $(p>0.05)$. No significant differences $(p>0.05)$ were also observed between the three groups in terms of the average of blood pressure and heart rate before surgery, and were almost the same in all three groups (Table 2.). According to Table 3, the mean duration of analgesia after surgery showed a significant difference in the three groups. As matter of fact, the average duration of analgesia in tramadol groups was greater than neostigmine group, as well as it was higher in neostigmine group compared with the placebo group $(p=0.01)$. The average pain score was zero in both tramadol and neostigmine groups on recovery. The mean pain score in the recovery of the placebo group was 3.1 times. Pain score in the recovery of the placebo group was higher compared with other groups. As shown in Table 4-4, the average pain score at 2 hours after surgery showed significant differences in the three groups. In fact, pain score in the placebo group was more than neostigmine group 2 hours after the operation. Furthermore, it was higher in neostigmine group compared with tramadol group $(p=0.01)$. There was no significant differences between neostigmine and tramadol groups regarding the mean pain score at 6 hours after surgery ( $p>0.05)$. But the pain score in the placebo group was higher than other groups $(\mathrm{p}<0.05)$.

According to Table 4-6, the average pain score 12 hours after surgery in the placebo group was more than other groups, while pain score in neostigmine group was also more than tramadol group $(\mathrm{p}=0.01)$. No significant difference was observed between the average blood pressure and heart rate recovery in the three groups of patients, almost all three groups showed similar outcomes $(\mathrm{P} \geq 0.05)$. There was no significant difference between the three groups in terms of the average blood

Table 2. Comparison of the mean blood pressure and heart rate in the patients undergoing preoperative Colporrhaphy in the three groups of Tramadol, Neostigmine and placebo.

Group

Tramadol

Neostigmine

placebo

$P$ value

Mean \pm SD

Preoperative pressure

$79.9 \pm 3.2$

$80.1 \pm 4.2$

$81.2 \pm 2.8$

$\mathrm{P} \geq 0.05$

Heart rate84.8 \pm 3.3

$83.5 \pm 1.8$

$85.2 \pm 2.2$

$\mathrm{P} \geq 0.05$ 


\section{Neostigmine, Tramadol and Lidocaine for post-operative pain control}

Eur J Transl Myol 28 (2): 185-192, 2018

Table 3. Comparisonof the mean duration of postoperative analgesia in patients undergoing Colporrhaphy, in the three groups of Tramadol, Neostigmine and placebo.

\begin{tabular}{|c|c|c|c|c|}
\hline \multirow[t]{2}{*}{ Group } & Tramadol & Neostigmine & placebo & \multirow[t]{2}{*}{$P$ value } \\
\hline & & Mean \pm SD & & \\
\hline $\begin{array}{l}\text { The mean duration of } \\
\text { analgesia after surgery } \\
\text { (Min) }\end{array}$ & $246.6 \pm 9.1$ & $165.2 \pm 8.3$ & $110.2 \pm 8.8$ & 0.01 \\
\hline
\end{tabular}

Table 4. Comparison of the average pain score 2 hours after surgery in patients undergoing Colporrhaphy, in the three groups Tramadol, Neostigmine and placebo.

\begin{tabular}{|c|c|c|c|c|}
\hline Group & Tramadol & Neostigmine & placebo & $P$ value \\
\hline $\begin{array}{l}\text { The mean pain } \\
\text { score at } 2 \text { hours } \\
\text { after surgery }\end{array}$ & $0.94 \pm 0.38$ & $1.5 \pm 0.85$ & $2.6 \pm 0.78$ & 0.01 \\
\hline
\end{tabular}

Table 5. Comparison of the average pain score in patients undergoing Colporrhaphy at 6 hours after surgery in Tramadol, Neostigmine and placebo groups.

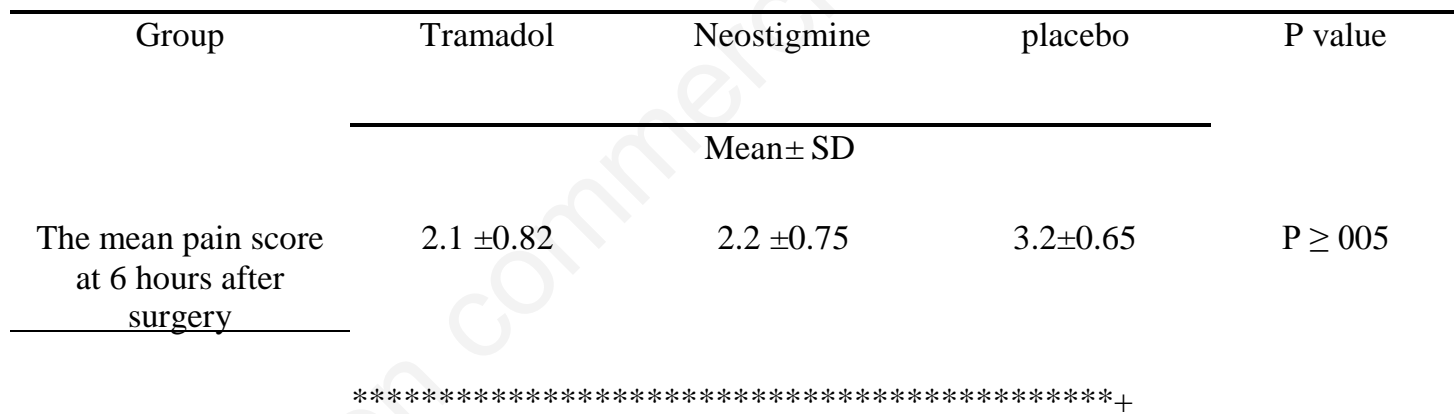

Table 6. Comparison of the average pain score in patients undergoing colporrhaphy, in tramadol, neostigmine and placebo groups, 12 hours after surgery.

\begin{tabular}{|c|c|c|c|c|}
\hline Group & Tramadol & Neostigmine & placebo & $P$ value \\
\hline & & Mean \pm SD & & \\
\hline $\begin{array}{c}\text { The mean pain } \\
\text { score at } 12 \text { hours } \\
\text { after surgery }\end{array}$ & $2.2 \pm 0.81$ & $3.4 \pm 0.95$ & $5.2 \pm 1.1$ & 0.01 \\
\hline
\end{tabular}

pressure and heart rate, 2 hours after surgery. As matter of fact, all three groups showed similar outcomes $(\mathrm{P} \geq 0.05)$. There was no significant difference between the three groups regarding to the average blood pressure and heart rate of patients 6 hours after surgery. It should be noted that similar results were found in all three groups $(\mathrm{P} \geq 0.05)$. As summarized in Tables 4 to 10 , the average analgesic drug consumption in the placebo group 24 hours after surgery was more than other groups. While the average analgesic drug consumption in the neostigmine group was higher compared with the tramadol group, 24 hours after surgery $(\mathrm{p}=0.01)$. 


\section{Neostigmine, Tramadol and Lidocaine for post-operative pain control}

Eur J Transl Myol 28 (2): 185-192, 2018

Table 7. Comparison of the average blood pressure and heart rate of recovery in patients undergoing Colporrhaphy in the three Tramadol, Neostigmine and placebo groups

\begin{tabular}{ccccc}
\hline Group & Tramadol & Neostigmine & placebo & P value \\
\cline { 2 - 4 } & & Mean \pm SD & \\
BP recovery & $74.5 \pm 4.1$ & $75.5 \pm 4.5$ & $76.6 \pm 3.4$ & $\mathrm{P} \geq 0.05$ \\
Heart rate recovery & $80.2 \pm 4.3$ & $80 / 9 \pm 3 / 8$ & $80 / 5 \pm 4 / 8$ & $\mathrm{P} \geq 0.05$ \\
\hline
\end{tabular}

$$
* * * * * * * * * * * * * * * * * * * * * * * * * * * * * * * * * * * * * * * * * * * *+
$$

Table 8. Comparison of the blood pressure and heart rate in the three groups of patients undergoing Colporrhaphy, 2 hours after surgery in Tramadol, Neostigmine and placebo groups.

\begin{tabular}{ccccc}
\hline Group & Tramadol & Neostigmine & placebo & P value \\
\cline { 2 - 4 } & & Mean \pm SD & \\
Blood pressure 2 hours after \\
$\begin{array}{c}\text { surgery } \\
\text { HR 2 hours after surgery }\end{array}$ & $83.3 \pm 2.8$ & $82.5 \pm 3.3$ & $84.5 \pm 4 / 5$ & $\mathrm{P} \geq 0.05$ \\
\hline
\end{tabular}

$$
* * * * * * * * * * * * * * * * * * * * * * * * * * * * * * * * * * * * * * * * * * * *+
$$

Table 9. Comparison of the average blood pressure and heart rate in the three groups of patients undergoing

\begin{tabular}{|c|c|c|c|c|}
\hline Group & Tramadol & Neostigmine & placebo & $\mathrm{P}$ value \\
\hline & & Mean \pm SD & & \\
\hline $\begin{array}{c}\text { Blood pressure } 6 \text { hours after } \\
\text { surgery }\end{array}$ & $81.1 \pm 4.2$ & $79.9 \pm 3.1$ & $80.1 \pm 2.1$ & $\mathrm{P} \geq 0.05$ \\
\hline HR 6 hours after surgery & $86.8 \pm 4.8$ & $85.1 \pm 3.4$ & $84.8 \pm 2.3$ & $\mathrm{P} \geq 0.05$ \\
\hline
\end{tabular}
Colporrhaphy 6 hours after surgery in Tramadol, Neostigmine and placebo groups.

$* * * * * * * * * * * * * * * * * * * * * * * * * * * * * * * * * * * * * * * * * * * *+$

Table 10. Comparison of the average analgesic drug (in $\mathrm{mg}$ ), 24 hours after surgery in the three tramadol,

\begin{tabular}{|c|c|c|c|c|}
\hline \multirow[t]{2}{*}{ Group } & Tramadol & Neostigmine & placebo & $\mathrm{P}$ value \\
\hline & & Mean \pm SD & & \\
\hline Average analgesic $(\mathrm{Mg})$ & $95.5 \pm 3.3$ & $145.4 \pm 4.5$ & $200.1 \pm 5.1$ & 0.01 \\
\hline
\end{tabular}
neostigmine and placebo groups.

Achieving the right drug combination to control postoperative pain in patients undergoing colporrhaphy surgery is one of the important goals in colporrhaphy surgery. Better and longer control of pain, by using new compounds in paracervical block, increased the level of satisfaction and reduced the side effects of surgery and 
anesthesia in patients. In this study, we compared the effects of adding tramadol and neostigmine to lidocaine $1.5 \%$ and postoperative pain management in patients. The results showed that tramadol had a greater and better effect in increasing analgesia for patients. The results obtained in this study are consistent with previous similar studies, so that in a study by Kamali et al., in 2016 on curettage candidates in which the effect of adding fentanyl and ketamine to lidocaine $1.5 \%$ in increasing the duration of analgesia in patients by using paracervical block were examined, it was found that both fentanyl and ketamine led to an increase in the duration of analgesia and increased quality of block, but no significant difference was observed between the two groups of ketamine and fentanyl in terms of pain score, while the duration of analgesia in the ketamine group was higher. ${ }^{22}$ In another study by Subedi et al., in 2013 on patients undergoing cesarean section, it was found that the addition of intrathecal tramadol and intrathecal fentanyl to bupivacaine $0.5 \%$ increase of the duration of analgesia and the quality of block, but no significant difference was observed between the two adjuvants. ${ }^{23}$ In another study by Kamali et al., in 2012 on patients undergoing colporrhaphy, 60 patients undergoing colporrhaphy were divided into two equal groups: neostigmine and midazolam and it was found that adding midazolam and neostigmine to $5 \%$ lidocaine by spinal method is effective in the duration of analgesia of patient, while the duration of analgesia in the midazolam group was significantly higher than neostigmine group $(\mathrm{p}<0.001)$. The rate of drug consumption during 24 hours in the midazolam group was lower than that of neostigmine. ${ }^{24}$ In another study by Taheri et al., in 2010 in children undergoing inguinal hernia surgery, the effect of adding neostigmine and tramadol to bupivacaine $0.25 \%$ was compared and it was found that adding both adjuvants led to a significant increase in the duration of anesthesia and score pain reduction in children, on the other hand, the duration of analgesia was higher in the tramadol group and the need for pain reliever in tramadol group was reported to be lower. ${ }^{25}$ In another study by Kamali et al. $(2010)^{26}$ in children of 2 to 8 year old, undergoing lower abdominal surgery, it was showed that the addition of neostigmine and midazolam to bupivacaine $0.25 \%$ in caudal anesthesia method, led to an increase in the duration of analgesia in children and improved quality of the block, while the duration of analgesia in the neostigmine group was more than in the midazolam group. In another study by Kamali et al. $(2015)^{27}$ on patients undergoing cesarean section, the effects of adding midazolam and tramadol to lidocaine $5 \%$ was compared. In this study it was found that the addition of both adjuvants (tramadol and midazolam) led to an increase in the duration of analgesia and improve quality of block, also pain score in patients and the drugs used in these patients within 24 hours after surgery in tramadol group was lower than midazolam group. The duration of analgesia in tramadol group was reported greater than midazolam group. In another study by Kappa et al. $(2003)^{28}$ on patients undergoing cesarean section, it was found that the addition of neostigmine and fentanyl to $0.5 \%$ bupivacaine led to an increase in the duration of analgesia, this is while the pain score and average narcotic use in 24 hours after surgery in the fentanyl group was lower than that of neostigmine. Results of previous studies show that both neostigmine and tramadol as adjuvants in many regional methods (spinal - epidural - caudal - paracervical block, etc.,) lead to an increase in the duration of analgesia and improved quality of the blocks. Results obtained in our study is consistent with a large number of similar previous studies and like previous studies, both adjuvants, tramadol and neostigmine, were effective in the increased duration of postoperative analgesia in patients, also like several previous studies, the effect of tramadol in increasing the duration of postoperative analgesia in patients and increased block quality was more than neostigmine. In this study, it is recommended to use both neostigmine and tramadol as adjuvants because of their effectiveness in improving the quality of blocks and the better effect of tramadol. Anyhow, it will be prudent to compare in future studies the effect of adding the two drugs to local anesthetics for paracervical block in more cases and in other women surgeries.

In conclusion, our data suggest that tramadol could reduce pain at 2, 6 and 12 hours after surgery and increase duration of analgesia. We also recommend to extend the use of these drugs in other cases of regional and local block methods.

\section{List of acronyms}

ASA - Anesthesiologists

ECG - Electrocardiography

ET $\mathrm{CO}_{2}$ - End-tidal $\mathrm{CO}_{2}$

NMDA - N-methyl-D-aspartate

MAC - Minimum Alveolar Concentration

\section{Author's contributions}

$\mathrm{AK}, \mathrm{BY}$ and $\mathrm{AKH}$ carried out the data collection, performed the statistical analyses and drafted the manuscript and they participated in the design of the study and MSH conducted writing and design. All authors read and approved the final manuscript.

\section{Acknowledgments}

There are no acknowledgments.

\section{Conflict of Interest}

The authors report no conflicts of interests.

\section{Ethical Publication Statement}

We confirm that we have read the Journal's position on issues involved in ethical publication and affirm that this report is consistent with those guidelines. 


\section{Neostigmine, Tramadol and Lidocaine for post-operative pain control}

Eur J Transl Myol 28 (2): 185-192, 2018

\section{Corresponding Author}

Maryam Shokrpour, Department of Gynecology, Arak University of Medical Sciences, Arak, Iran. Phone: 00989181622810

Email: Maryam_shokrpour@yahoo.com

E-mails of co-authors

Alireza Kamali: Alikamaliir@yahoo.com

Bijan Yazdi: yazdi@arakmu.ac.ir

Atefeh Khalilpour: khalilpoor1384@yahoo.com

\section{References}

1. Lamers BH, Broekman BM, Milani AL. Pessary treatment for pelvic organ prolapse and healthrelated quality of life: a review. Int Urogynecol J 2011;22:637-44.

2. Jelovsek J, Maher C, Barber M. Prolaps. Cleveland Clinic: Euclid Avenue 2007.

3. Altman D, Väyrynen T, Engh ME, et al. Nordic Transvaginal Mesh Group. Anterior colporrhaphy versus transvaginal mesh for pelvic-organ prolapse. N Engl J Med 2011;364:1826-36.

4. Cespedes R, Cross K, Gurie E. Pelvic prolapse: Diagnosing \& Treating cyctocele, rectocele. Medscape Womens Health 1998 ;3:4.

5. Hawkins JL, Gibbs CP, Orleans M, Martin-Salvaj G, Beaty B. Obstetric anesthesia work force survey, 1981 versus 1992. Anesthesiology 1997;87:135-43.

6. Ameres M, Yeh B. Pain after surgery. Emedicine health 2011.

7. Kehlet H, Holte K. Effect of postoperative analgesia on surgical outcome. Br J Anaesth 2001;87:62-72.

8. Julius D, Basbaum AI. Molecular mechanisms of nociception. Nature 2001;413:203-10.

9. Son A, Rudra A, Sarkar S, Biswas B. Intrathecal midazolam for postoperative pain relief in cs. J Indian Med Assoc 2001:683-6.

10. Siddiqi R, Jafri SA. Maternal satisfaction after spinal anesthesia for c/s. J Coll Physicians Surg Pak 2009; 19:77-80

11. Liu S, Carpenter RL, Neal JM. Epidural anesthesia and analgesia. Their role in postoperative outcome. Anesthesiology 1995;82:1474-506.

12. Chanrachakul B, Likitanasombut P, OPrasertsawat P, Herbutya Y. Lidocaine versus plain saline for pain relief in fractional curettage : A randomized controlled trial. Obstet Gynecol 2001;98:592-5.

13. Mankowski JL, Kingston J, Moran T, et al. Paracervical compared with intracervical lidocaine for suction curettage : A Randomized controlled Trial. Obstet Gynecol 2009;113:1052-7.

14. Rogers R. Chronic pelvic pain : An integrated approach. In: Steege J, Metzger D, Levy B, eds. Basic pelvic neuroanatomy. Philadelphia: Saunders company 1998:31-58.
15. Rattanachaiyanont $M$, Leerasiri $P$, Indhavivadhana S. Effectiveness of intrauterine anesthesia for pain relief during fractional curettage. Obstet Gynecol 2005;106:533-9.

16. Ejlersen E, Andersen HB, Eliasen K, Mogensen T. A comparison between preincisional and postincisional lidocaine infiltration and postoperative pain. Anesth Analg 1992;74:495-8.

17. Honarmand A, Safavi MR. Comparison of prophylactic use of midazolam, ketamine, and ketamine plus midazolam for prevention of shivering during regional anaesthesia: a randomized double-blind placebo controlled trial. Br J Anaesth. 2008;101:557-62.

18. Mahmood MA, Zweifler RM. Progress in shivering control. J Neurol Sci 2007;261:47-54.

19. Crowley LJ, Buggy DJ. Shivering and neuraxial anesthesia. Reg Anesth Pain Med 2008;33:241-52.

20. Pang WW, Huang PY, Chang DP, Huang MH. The peripheral analgesic effect of tramadol in reducing propofol injection pain: a comparison with lidocaine. Reg Anesth Pain Med 1999;24:246-9.

21. Edwards JE, McQuay HJ, Moore RA. Combination analgesic efficacy: individual patient data metaanalysis of single-dose oral tramadol plus acetaminophen in acute postoperative pain. J Pain Symptom Manage 2002;23:121-130.

22. Kamali AR, Shokrpour M, Jamilian M, et al. Comparision of addition Fentanyl and Ketamin to $1 / 5 \%$ Lidocain in paracervical block for reducing of post operative pain in curettage. Biom Pharmaco J 2016;9:949-55.

23. Subedi A, Biswas BK, Tripathi M, Bhattarai BK, Pokharel K. Analgesic effects of intrathecal tramadol in patients undergoing caesarean section: a randomised, double-blind study. International Journal of Obstetric Anesthesia 2013;22:316-21.

24. Kamali A, Pazoki S, Shokrpour M, Vatanpour K. Comparison of Effect of Additive midazolam with Neostigmine to lidocaine 5\% in Post operation pain in colporrhaphy surgery in Spinal Anesthesia. Arak Medical University Journal 2012;15:35-41.

25. Taheri R, Shayeghi S, Razavi SS, et al. Efficacy of bupivacaine-neostigmine and bupivacainetramadol in caudal block in pediatric inguinal herniorrhaphy. Paediatric anaesthesia 2010;20:866-72.

26. Davoudi M, Kamali AR.A comparison of caudal anesthesia with midazolam and neostigmin co administered with bupivacain in reduction of postoperative pain following lower abdominal surgery in pediatrics (2-8 years old). Arak Medical U J 2011:14;17-23.

27. Pazuki S, Kamali A, Shahrokhi N, Jamilian M. Comparison of the Effects of Intrathecal Midazolam and Tramadol with the Conventional 


\section{Neostigmine, Tramadol and Lidocaine for post-operative pain control}

Eur J Transl Myol 28 (2): 185-192, 2018

Method of Postoperative Pain and Shivering Control after Elective Cesarean Section. Biom Pharmaco J 2016: 9;995-1003.
28. Kaya FN, Sahin S, Owen MD, Eisenach JC. Epidural neostigmine produces analgesia but also sedation in women after cesarean delivery. Anesthesiology 2004;100:381-5.

Submission: $16 / 02 / 18$

Revisions received: 20/02/18

Acceptance: $21 / 02 / 18$ 